\title{
Thermoelastic stresses and defect production in semicon- ductor-insulator structures at isothermic heating
}

\author{
O.A. Agueev and A.M. Svetlichnyi \\ Taganrog State Radio Engineering University, 44 Nekrasovskii per., GSP-17A, Taganrog, Rostov Region, Russia \\ Tel.: (863-44)6-16-11,E-mail: svetlich@tsure.ru
}

\begin{abstract}
For semiconductor structure substrates with film coating disturbances we investigated thermoelastic stresses and their effect on defect production at isothermic heating. A developed mathematical model enables one to optimize the formation and annealing conditions for structures with film coating disturbances during annealing when manufacturing integrated microcircuits.
\end{abstract}

Keywords: semiconductor substrates, film coatings, isothermic heating, thermoelastic stresses, defect production, dislocations.

Paper received 03.12.99; revised manuscript received 08.05.00; accepted for publication 30.06.00.

\section{Introduction}

One of the lines of advancement in microelectronic technologies is increasing the scale of integration for microcircuits. In this case various spatial nonuniformities and structural defects of the substrate material become comparable in size to the integrated microcircuit (IC) elements, and so have a pronounced effect on the operation of the latter.

ICs incorporate a great number of layers. Among them one may recognize semiconductor substrates, epitaxial structures, isolating layers, conducting layers, etc. An important peculiarity of the IC structures design is complex configuration of the layers that differ substantially in their physico-mechanical characteristics. To illustrate, for silicon substrate, conducting strips, silicon dioxide films and silicon nitride films the Young's modulus $(E)$ values vary from $2 \cdot 10^{10}$ to $1.7 \cdot 10^{12} \mathrm{dyne} / \mathrm{cm}^{2}$, the Poisson's ratio $(v)$ values differ by a factor of two, and the coefficient of linear expansion $\left(\alpha_{T}\right)$ values lie between $0.5 \cdot 10^{-6}$ and $4.2 \cdot 10^{-6} \mathrm{~K}^{-1}$.

The above facts indicate that nonuniform stress fields may appear in the ICs structures. These fields may have a pronounced effect on the parameters of IC active elements, as well as promote defect formation in the crystal structures. That is why the problems related to both defect production and stresses in the IC structures attract more and more attention of the researchers engaged in the IC development and manufacturing [1-4]. The reason for this is that stresses substantially affect IC parameters, especially when the technological procedures are used that involve pulse laser, electron and photon beams [5-8].
When performing the present work, our objective was to develop a mathematical model describing both the stress field formation and defect production in the semiconductor substrate-insulator film structure having a typical for IC structures nonuniformity, namely, film coating disturbance.

\section{Stresses in IC structures}

Let us consider the conditions and reasons for stress appearance in the IC structures. The stress in a film, $\mathrm{s}_{\mathrm{f}}$, may be determined with the following expression:

$\sigma_{\mathrm{f}}=\sigma_{\mathrm{int}}+\sigma_{\mathrm{th}}$.

Here $\sigma_{\text {int }}$ is the stress that appeared during formation (it is determined by the film composition and formation conditions); $\sigma_{\mathrm{th}}$ is the thermoelastic stress that appeared during heat treatment (it is determined by the film properties and heat temperature).

The stress that appears during film formation is of intricate nature. It depends on the technique used to form the film, as well as on the formation conditions. For instance, in $\mathrm{SiO}_{2}$ films prepared using thermal oxidation of silicon substrate, the stress depends not only on the physico-chemical properties of the film and substrate but also on (i) the difference $\Delta T=T_{h}-T_{f}$ between the heat temperature, $T_{h}$, and film formation temperature, $T_{f}$ and (ii) the ratio between the molar volumes of $\mathrm{SiO}_{2}$ and silicon that has reacted with oxygen, $\gamma(\gamma=4 / 9$ [9]). In this case one can estimate the $\mathrm{s}_{\text {int }}$ value for $\mathrm{SiO}_{2}$ films using the following expression [9]: 


\section{O.A. Agueev, A.M. Svetlichnyi: Thermoelastic stresses and defect production in ...}

$\sigma_{\text {int }}=\frac{2(1-\gamma) \Delta T\left\lfloor\left(\mathrm{E}_{\mathrm{SiO}_{2}} / 1-v_{\mathrm{SiO}_{2}}\right) \alpha_{\mathrm{SiO}_{2}}+(1-\gamma)\left(\mathrm{E}_{\mathrm{Si}} / 1-v_{\mathrm{Si}}\right) \alpha_{\mathrm{Si}}\right\rfloor\left(\mathrm{E}_{\mathrm{Si}} / 1-v_{\mathrm{Si}}\right)}{\left(\mathrm{E}_{\mathrm{SiO}_{2}} / 1-v_{\mathrm{SiO}_{2}}\right)+(1-\gamma)\left(\mathrm{E}_{\mathrm{Si}} / 1-v_{\mathrm{Si}}\right)}$

To estimate the $\sigma_{\text {th }}$ value, one may use the expression given in [2]:

$$
\sigma_{\mathrm{th}}=\Delta \alpha_{T} T_{h} \frac{E_{\mathrm{SiO}_{2}}}{1-v_{\mathrm{SiO}_{2}}}
$$

Here $\Delta \alpha_{T}$ is the difference between the coefficients of linear expansion for the film and substrate.

The above stresses are distributed uniformly over the film thickness. The stresses in the substrate are the same in magnitude as those in the film but opposite in sign [2-4]. One has also to take into account that the microstresses appear in structures near kinks, jogs and edges of film coating disturbances (all the above disruptions serve as the stress concentrators). These microstresses substantially exceed stresses in the uniform (i.e., lying far from the edges of film coating disturbances) layer regions [2-4].

Due to spatial nonuniformity of the stress fields, it is rather difficult to analyze them. Therefore, one has to make various assumptions when deriving analytical relations. In particular, to estimate the effect of film edge on the substrate, an edge force is introduced [2]. It has two components: $f$ (directed along the substrate surface) and $y$ (directed normally to the substrate surface) $[1,3]$. Their values may be determined from the following expressions [1]:

$f=\sigma_{\mathrm{f}} h$

$\psi=\alpha \cdot f$

$\alpha=\sqrt{\frac{3 \cdot \sigma_{f}}{E_{f}}}$.

Here $E_{f}$ is Young's modulus of the film.

Let us consider a stress concentrator (see Fig. 1). It is a semiinfinite semiconductor substrate with an insulator film region where there is a disturbance. Let $x$ and $y$ axes

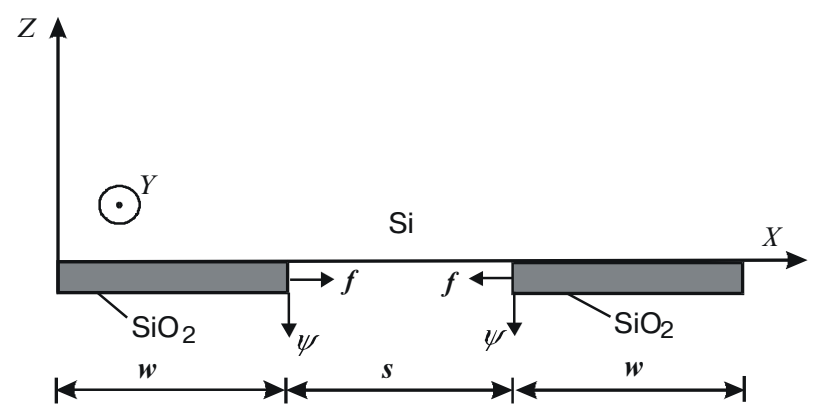

Fig. 1. A semiconductor substrate region (2) with an insulator film disturbance (1). be oriented along the substrate surface, $z$ axis be oriented to the substrate bulk, and $w$ be the width of the film region located on the substrate at a distance $\sigma$ from another region. The edge force components, $f$ and $\psi$, are oriented as was stated above. Then, for such a configuration, the substrate is plane-strained; the stress tensor components are as follows:

$$
\begin{aligned}
& \sigma_{x x}(x, z)=-\frac{2 f}{\pi} \cdot \frac{x^{2}}{\left(x^{2}+z^{2}\right)^{2}} \cdot(x+\alpha z) \\
& \sigma_{z z}(x, z)=-\frac{2 f}{\pi} \cdot \frac{z^{2}}{\left(x^{2}+z^{2}\right)^{2}} \cdot(x+\alpha z) \\
& \sigma_{z x}(x, z)=-\frac{2 f}{\pi} \cdot \frac{2 x}{\left(x^{2}+z^{2}\right)^{2}} \cdot(x+\alpha z)
\end{aligned}
$$

For an actual IC structure the concentrators are arranged periodically, their spacing being comparable to their size (e.g., after photolithography procedure and etching local regions in the insulator film). Then the structure presented in Fig. 1 is an elementary stress concentrator. A regular system involving $2 N$ elementary stress concentrators is shown in Fig. 2. In this case one may calculate the distribution of the stress tensor components in an isotropic substrate (with allowance made for the action of adjacent concentrators) using the expressions given in [2]:

$$
\begin{aligned}
& \sigma_{x x}(x, z)=-\frac{2 f}{2 \pi} \times \\
& \times \sum_{n=-N}^{N}\left(\begin{array}{l}
\frac{[x-n \cdot(w+s)]^{2} \cdot[x-n \cdot(w+s)+\alpha z]}{\left\{[x-n \cdot(w+s)]^{2}+z^{2}\right\}^{2}}- \\
-\frac{[x-n \cdot(w+s)-w]^{2} \cdot[x-n \cdot(w+s)-w-\alpha z]}{\left\{[x-n \cdot(w+s)-w]^{2}+z^{2}\right\}^{2}}
\end{array}\right)
\end{aligned}
$$

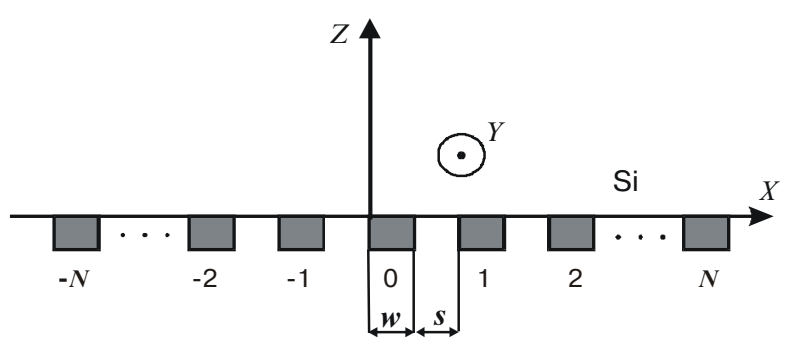

Fig. 2. A regular arrangement of stress concentrators. 
$\sigma_{z z}(x, z)=-\frac{2 f}{2 \pi} z^{2} \sum_{n=-N}^{N}\left(\begin{array}{c}\frac{x-n \cdot(w+s)+\alpha z}{\left\{[x-n \cdot(w+s)]^{2}+z^{2}\right\}^{2}}- \\ -\frac{x-n \cdot(w+s)-w-\alpha z}{\left\{[x-n \cdot(w+s)-w]^{2}+z^{2}\right\}^{2}}\end{array}\right)$

$\sigma_{z x}(x, z)=-\frac{2 f}{2 \pi} z \times$

$\times \sum_{n=-N}^{N}\left(\begin{array}{l}\frac{[x-n \cdot(w+s)] \cdot[x-n \cdot(w+s)+\alpha z]}{\left\{[x-n \cdot(w+s)]^{2}+z^{2}\right\}^{2}}- \\ -\frac{[x-n \cdot(w+s)-w] \cdot[x-n \cdot(w+s)-w-\alpha z]}{\left.\{x-n \cdot(w+s)-w]^{2}+z^{2}\right\}^{2}}\end{array}\right)$

\section{Defect production in substrate}

It is known that the physico-mechanical characteristics of semiconductor single crystals are anisotropic. This anisotropy has a pronounced effect on the defect production. In particular, for crystals with face-centered cubic lattice the dislocation-type defects are located in the $\{111\}$ crystallographic planes and are oriented in the $<110>$ directions. To determine the effect of stresses on defect production in such crystals, one has to find the resulting shear stresses, $\tau$.

Plastic deformation accompanied by the dislocation production occurs when the resulting shear stresses exceed some critical value, $\sigma_{E}$, at which plastic deformation begins to develop. This critical value depends on temperature and may be found from the following expression:

$\sigma_{E}(T)=H \varepsilon^{\frac{1}{n}} \exp \left(\frac{U}{n k T}\right)$

Here $H$ and $n$ are constants; $U[\mathrm{eV}]$ is the plastic deformation energy; $\varepsilon\left[\mathrm{s}^{-1}\right]$ is the deformation rate; $k=6.85 \cdot 10^{-5}$ $[\mathrm{eV} / \mathrm{K}]$ is the Boltzmann constant [2].

After introduction of the defect production criterion (it is defined as the ratio between $t$ and $\sigma_{E}, \tau / \sigma_{E}$ ) and plot- ting its distribution in the $(x, z)$ coordinates, one can determine the regions with $\tau / \sigma_{E}>1$ where dislocations are produced. The density of dislocations, $N_{\text {dis }}$, produced in the substrate region where $\tau / \sigma_{E}>1$ (at relaxation of the resulting shear stresses) may be estimated by solving the following equation in $N_{d i s}[10]$ :

$$
\frac{\tau}{\sigma}=\frac{2 b \tau}{K A^{2}} \cdot\left[\frac{A \sqrt{N_{0}}-A \sqrt{N_{d i s}}}{\tau}+\ln \left(\frac{1-\frac{A \sqrt{N_{0}}}{\tau}}{1-\frac{A \sqrt{N_{d i s}}}{\tau}}\right)\right] .
$$

Here $G$ is the shear modulus; $b$ is the Burgers vector; $N_{0}$ is the initial dislocation density in the crystal; $K=8 \cdot 10^{-7}$ dyne/cm is constant; $A=G b / 3$.

Thus, using the above mathematical model (expressions (1)-(7)), one can estimate the effect of heat treatment on defect production in structures, as well as optimize the formation and annealing conditions for the structures with film coating disturbances.

\section{Stresses in $\mathrm{Si}_{-} \mathrm{SiO}_{2}$ structures}

To illustrate, let us consider a silicon substrate with $\mathrm{SiO}_{2}$ layer (whose thickness $h$ is $1 \mu \mathrm{m}$ ) prepared using thermal oxidation at a temperature $T_{f}=1100{ }^{\circ} \mathrm{C}$.

Using the values of the physico-mechanical parameters for silicon and silicon dioxide given in the Table 1 and the expressions (1) $-(3)$, one can obtain that at $T_{h}=800{ }^{\circ} \mathrm{C}$ $\left(\Delta T=300{ }^{\circ} \mathrm{C}\right)$ the stresses in the $\mathrm{SiO}_{2}$ film are as follows: $\sigma_{\text {int }}=-2.1 \cdot 10^{9}$ dyne $/ \mathrm{cm}^{2} ; \sigma_{\text {th }}=2.55 \cdot 10^{9} \mathrm{dyne} / \mathrm{cm}^{2}$; $\sigma_{\mathrm{f}}=4.5 \cdot 10^{8} \mathrm{dyne} / \mathrm{cm}^{2}$. In this case the distribution of the tensor components $\sigma_{x x}, \sigma_{z z}$ and $\sigma_{z x}$ for the stress in substrate resulting from the elementary stress concentrator (Fig. 1) may be calculated using expression (5). Its form is shown in Fig. 3. The calculations were performed for the region with $w+s=180 \mu \mathrm{m}(w=s=90 \mu \mathrm{m})$ in steps of $(w+s) / 40=4.5 \mu \mathrm{m}, N=5$.

An analysis of the results of calculations evidences that they completely agree with the general rules discussed in [2-4], namely:

the stress tensor components are concentrated near the edge of the film coating disturbance;

the distribution of the stress tensor components is

Table 1. Physico-mechanical characteristics of some materials that are used in microelectronics [1].

\begin{tabular}{ccccc}
\hline \hline Material & Applications & $\begin{array}{c}\text { Young's modulus } \\
E, \text { dyne/cm }\end{array}$ & Poisson's ratio $v$ & $\begin{array}{c}\text { Coefficient of linear } \\
\text { expansion } \alpha_{T}, 10^{-6} \mathrm{~K}^{-1}\end{array}$ \\
\hline $\mathrm{Si}$ & substrate & $1.7 \cdot 10^{12}$ & 0.27 & 2.2 \\
\hline $\mathrm{Al}$ & $\begin{array}{c}\text { conductor strips, } \\
\text { bonding islands }\end{array}$ & $6.5 \cdot 10^{11}$ & 0.35 & 2.5 \\
\hline $\mathrm{SiO}_{2}$ & insulation, gate insulator & $0.72 \cdot 10^{12}$ & 0.17 & 0.5 \\
\hline $\mathrm{Si}_{3} \mathrm{~N}_{4}$ & insulation, masking layers & $0.2 \cdot 10^{11}$ & - & $2.25-2.9$ \\
\hline \hline
\end{tabular}




\section{O.A. Agueev, A.M. Svetlichnyi: Thermoelastic stresses and defect production in ...}

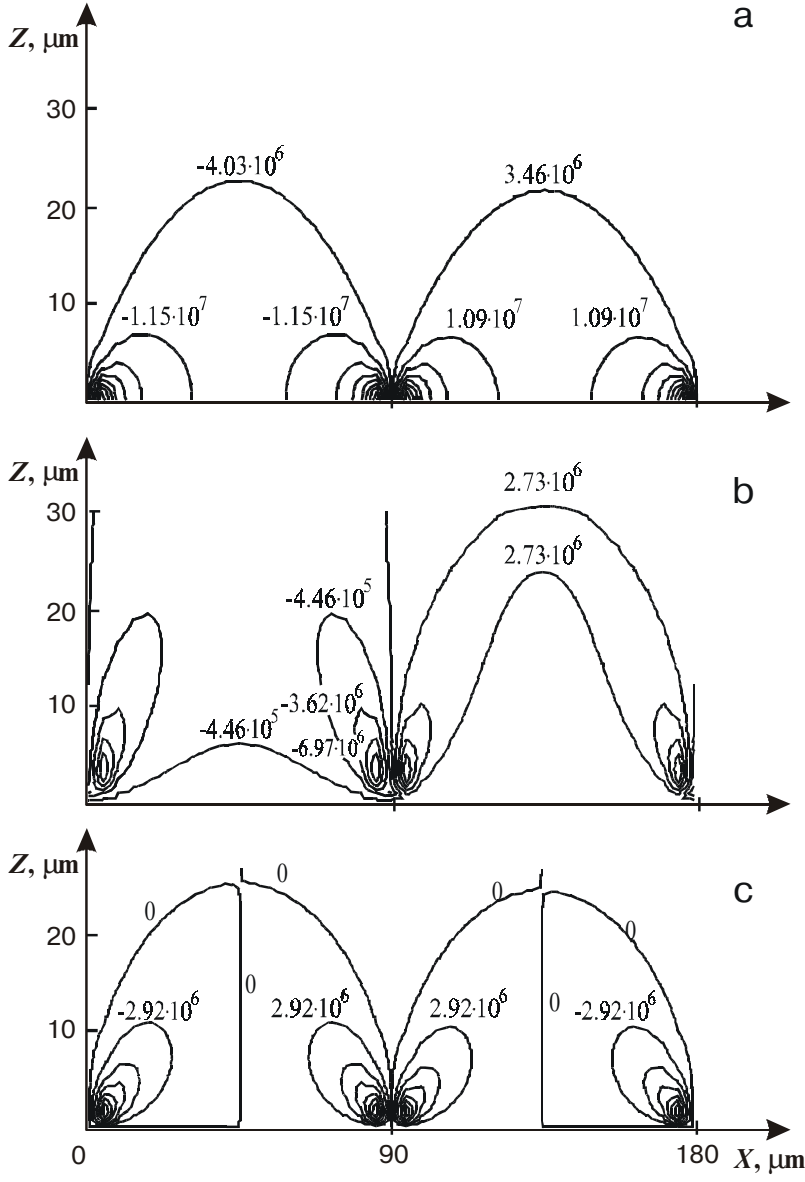

Fig. 3. Distribution of the stress tensor components $\left[\mathrm{dyne} / \mathrm{cm}^{2}\right]$ in the substrate with an elementary stress concentrator (shown in Fig. 2): $\mathrm{a}-\sigma_{x x} ; \mathrm{b}-\sigma_{z z} ; \mathrm{c}-\sigma_{x z}$.

symmetric about the plane passing through the edge of the film coating disturbance;

- the stress tensor components reverse their sign at the edge of the film coating disturbance.

If the $\mathrm{SiO}_{2}$ film edge is aligned with the $<110>$ azimuth at the (111)-oriented Si substrate, then the resulting shear stress may be determined from the following expression [11]:

$\tau=\sigma_{x x}-\frac{2}{3} \sigma_{z z}+\sqrt{3} \sigma_{z x}$

For the case of an elementary stress concentrator (Fig. 1) the calculated (with the above expression) resulting shear stress distribution in the Si substrate is presented in Fig. 4 on an enlarged scale, while the distribution of the defect production criterion is shown in Fig. 5. When calculating the critical stress value $\sigma_{E}$, the following parameter values were used: $H=4.5 \cdot 10^{5}\left[\mathrm{dyne} / \mathrm{cm}^{2}\right]$, $n=2.9, U=2.3[\mathrm{eV}], \varepsilon=9 \cdot 10^{-5}\left[\mathrm{~s}^{-1}\right]$.

An analysis of the distribution of the defect production criterion evidences that, under the considered heat treatment conditions and structure geometry, there exist

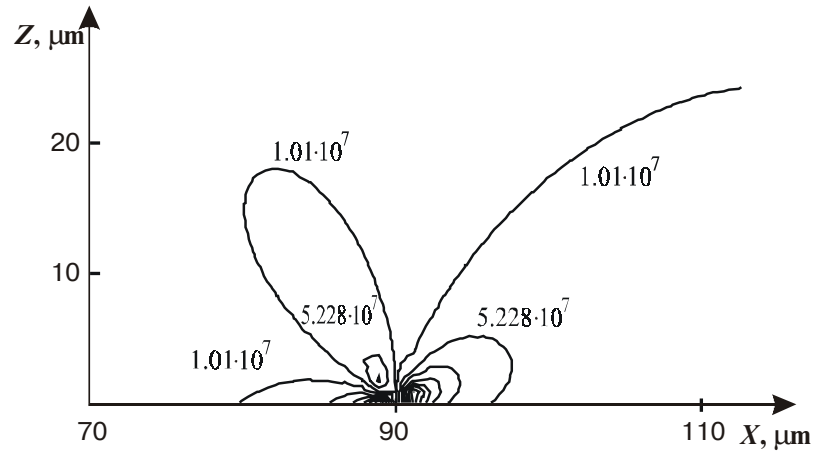

Fig. 4. Distribution of the resulting shear stresses $\left[\mathrm{dyne} / \mathrm{cm}^{2}\right]$ in the substrate with an elementary stress concentrator (shown in Fig. 1).

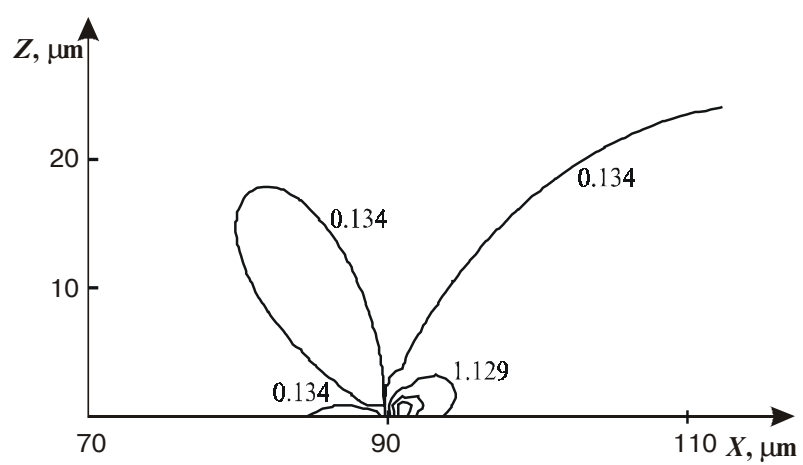

Fig. 5. Distribution of the defect production criterion in the substrate with an elementary stress concentrator (shown in Fig. 1).

the substrate regions where the resulting shear stresses exceed the critical value, and so dislocations are produced there. These regions lie near the Si substrate surface that is free from $\mathrm{SiO}_{2}$. Such a conclusion is confirmed by the experimental results [12]. According to them, oxide films increase the critical stress in the substrate by $50-100 \%$. This is due to healing of submicrocracks and slowing down the dislocation movement.

An estimation of dislocation density made using expression (7) gives $N_{\text {dis }}=4.14 \cdot 10^{6} \mathrm{~cm}^{-2}$. When performing the estimation, $\tau$ was taken to equal $\tau_{\max }$ (i.e., the maximum value of the resulting shear stresses), $b=2.7 \cdot 10^{-8} \mathrm{~cm}$, $N_{0}=10^{2} \mathrm{~cm}^{-2}$.

\section{Conclusion}

The presented mathematical model enables one to calculate the thermoelastic stress distribution in the semiconductor-insulator structures. Through comparison between the distribution obtained and the critical stress value it is possible to evaluate the effect of heat treatment on defect production in the semiconductor substrates. The 


\section{O.A. Agueev, A.M. Svetlichnyi: Thermoelastic stresses and defect production in ...}

proposed model may be used for optimization of the heat treatment conditions to reduce the defect concentration in substrates, as well as at formation of local dislocation structures.

\section{References}

1. N.P. Zakharov, A.V. Bogdasaryan, Mechanical Phenomena in Integrated Structures, Radio i Svyaz', Moscow (1992).

2. S.M. Hu, Stress-related problem in silicon technology // J. Appl. Phys. 70, p. R53 (1991).

3. J. Vanhellemont, S. Amelinckx, Film-edge-induced dislocation generation in silicon substrates // J. Appl. Phys. 61, p. 2170 (1987).

4. S.C. Jain, H.E. Maes, K. Pinardi, I. De Wolf, Stresses and strains in lattice-mismatched stripes, quantum wires, quantum dots, and substrates in Si technology // J. Appl. Phys. 79, p. 8145 (1996).

5. D.A. Sechenov, A.M. Svetlichnyi, S.I. Solov'ev, O.A. Ageev, Effect of heating rate on the thermal stresses appearance in a silicon wafer at rapid annealing // Fiz. Khim. Obrab. Mater. № 5, p. 46 (1992).
6. D.A. Sechenov, A.M. Svetlichnyi, O.A. Ageev, A.G. Klovo, Simulation of temperature fields in semiconductor structures at rapid heat annealing // Fiz. Khim. Obrab. Mater. № 2, p. 33 (1994).

7. D.A. Sechenov, A.M. Svetlichnyi, A.G. Garitsyn, S.I. Solov'ev, O.A. Ageev, Simulation of semiconductor structures heating with laser radiation // Fiz. Khim. Obrab. Mater. № 2, p. 109 (1995).

8. D.A. Sechenov, A.M. Svetlichnyi, O.A. Ageev, Temperature and stress distributions in the STM tip influence area in silicon // Izvestiya Vuzov. Elektronika № 3, p. 52 (1998).

9. H.C. Liu, S.P. Murarka, Elastic and viscoelastic analysis of stress in thin films // J. Appl. Phys. 72, p. 3458 (1992).

10. P. Haasen, Dislocation dynamics in the diamond structure, in Dislocation Dynamics, Eds. A.R. Rosenfield, C.T. Hahv, A.L. Bement, R.I. Jaffe, p. 701, McGraw-Hill Book Company (1967).

11. A. Fischer, Calculation of the shear stress in (111)- and (001) semiconductor substrates with film edges // Crystal Res. \& Technol. 20, p. 65 (1985).

12. M.Kh. Shorshorov, V.P. Alekhin, Effect of environment and surface condition on plastic deformation in crystals (A review) // Fiz. Khim. Obrab. Mater. № 1, p. 61 (1976). 